\title{
Role of Jun N-terminal Kinase (JNK) signaling in the wound healing and regeneration of a Drosophila melanogaster wing imaginal disc
}

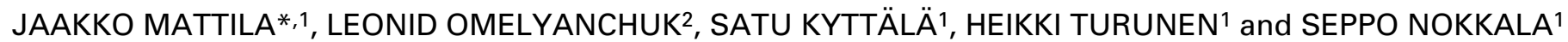 \\ ${ }^{1}$ Laboratory of Genetics, Department of Biology, University of Turku, Finland and \\ ${ }^{2}$ Laboratory of Cell Cycle Genetics, Institute of Cytology and Genetics, Novosibirsk, Russia
}

\begin{abstract}
When a fragment of a Drosophila imaginal disc is cultured in growth permissive conditions, it either regenerates the missing structures or duplicates the pattern present in the fragment. This kind of pattern regulation is known to be epimorphic, i.e. the new pattern is generated by proliferation in a specialized tissue called the blastema. Pattern regulation is accompanied by the healing of the cut surfaces restoring the continuous epithelia. Wound healing has been considered to be the inductive signal to commence regenerative cell divisions. Although the general outlines of the proliferation dynamics in a regenerating imaginal disc blastema have been well studied, little is known about the mechanisms driving cells into the regenerative cell cycles. In this study, we have investigated the role of Jun $\mathbf{N}$-terminal Kinase (JNK) signaling in the wound healing and regeneration of a Drosophila wing imaginal disc. By utilizing in vivo and in vitro culturing of incised and fragmented discs, we have been able to visualize the dynamics in cellular architecture and gene expression involved in the healing and regeneration process. Our results directly show that homotypic wound healing is not a prerequisite for regenerative cell divisions. We also show that JNK signaling participates in imaginal disc wound healing and is regulated by the physical dynamics of the process, as well as in recruiting cells into the regenerative cell cycles. A model describing the determination of blastema size is discussed.
\end{abstract}

KEY WORDS: wing imaginal disc, regeneration, wound healing, Jun N-terminal Kinase (JNK)

\section{Introduction}

The imaginal discs of Drosophila melanogaster have an intriguing capability to regenerate missing structures. When cut into two pieces and cultured in the abdomens of adult flies, in general the larger piece undergoes regeneration while the complementary piece duplicates (Bryant 1971, Schubiger 1971). An absolute requirement for pattern regulation (regeneration/duplication) to occur is growth (Schubiger 1973). In particular, pattern regulation of an imaginal disc is known to be epimorphic (Dale \& Bownes 1980, Bryant \& Fraser 1988) (although suggestions to the contrary exist, see Adler 1981), i.e. the emerging of the new pattern is dependent on cell divisions taking place in a specialized proliferating tissue called the blastema. The dynamics of regenerative proliferation have been extensively studied by clonal analysis (Abbot et al. 1981), incorporation of ${ }^{3} \mathrm{H}$-thymidine into replicating cells (Dale \& Bownes 1980, 1981, Adler 1984, Kiehle \& Schubiger 1985, O'Brochta \& Bryant 1987), direct cell counting
(Dunne 1981), volume measurement of cultured disc fragments (Adler 1981), flow cytometric analysis (Fain \& Alvarez 1987) and immunolocalization of incorporated bromodeoxyuridine (Bryant \& Fraser 1988).

Regeneration of an imaginal disc is accompanied by the healing of the cut edge surfaces, first by transient heterotypic contacts of the peripodial membrane and columnar epithelia and later by homotypic healing as the cell layers fuse to restore the continuous epithelia (Reinhardt et al. 1977, Reinhardt \& Bryant 1981). The prevailing view of pattern regulation is that apposition of differential positional values by homotypic healing induces intercalary growth resulting in either regeneration or duplication depending on the developmental capacity of the apposed cells. This idea, largely based on the polar co-ordinate model developed by French et al. (1976), is however impaired by the finding

Abbreviations used in this paper: GFP, green fluorescent protein; JNK, Jun Nterminal Kinase.

\footnotetext{
*Address correspondence to: Jaakko Mattila. Institute of Biotechnology, Viikinkaari 9 (P.O. Pox 56), 00014 University of Helsinki, Finland. Fax: +358-9-19159366. e-mail: jaakko.mattila@helsinki.fi
} 
A

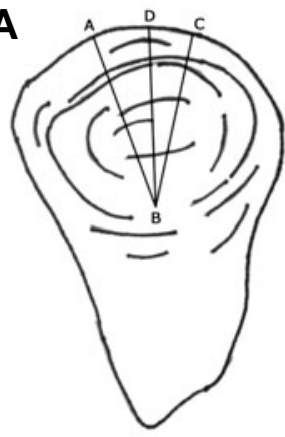

B

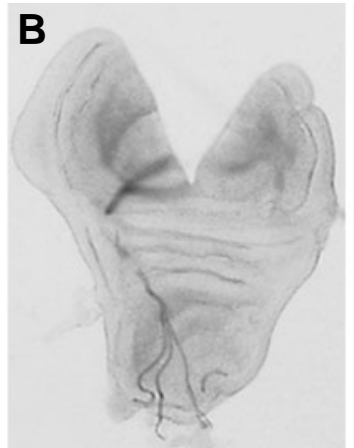

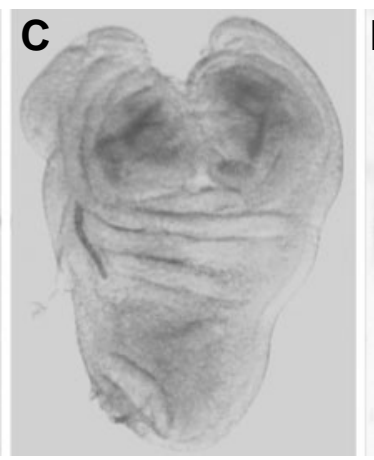

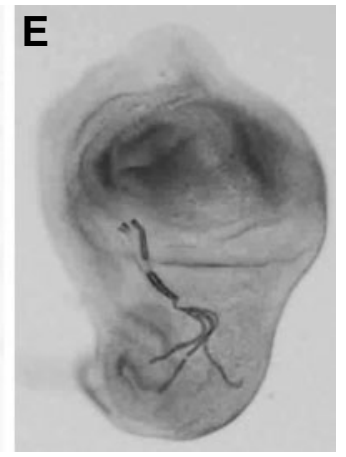

Fig. 1. The cuts used in the study and the classification of the wound healing process. (A) An incisional cut along the wing proper ( $D$ ' $B$ ) was utilized in the wound healing experiments, i.e. live confocal imaging and monitoring of the JNK signaling dynamics. To observe the proliferation pattern during regeneration, a $30^{\circ}$ wedge-shaped piece was removed from the wing pouch ( $A^{\prime} B^{\prime} C$ cut). The cuts were performed using a fine tungsten needle. (B-E) Representatives of the healing phase classes used in the study. (B) The disc is unhealed (in the picture) or shows some initial contraction of the wound edges towards each other. (C) The wound is closed half of its length. (D) The wound is closed some 3/4 of its length. A clear "notch" is visible in the site of the cut. (E) The wound is wholly closed and a continuous epithelium is reformed.

that, during normal development, discontinuity in cell fates does not result in additional growth (Chen \& Struhl 1996, see Held 2002 for discussion) and further, that regeneration from an L1 anterior $1 / 4$ fragment is dependent on the Hedgehog signal from the peripodial membrane rather than the interaction between the wound edges (Gibson \& Schubiger 1999), the latter being in favour of the boundary model of Meinhardt (1983). DNA synthesis, induced by the disc fragmentation, has been clearly shown to precede homotypic wound healing (Dale \& Bownes 1980, 1981, Bryant \& Fraser 1988) and consequently it has been suggested that the cells next to the cut edge would arrest in G2 until their apposition by wound healing triggers the cells to enter mitosis (Fain \& Alvarez 1987). However, at present it is not known how the cells near the cut edges are recruited into the cell cycle, nor is it even directly shown whether the cell divisions are regulated by the wound healing process.

The Jun N-terminal Kinase (JNK) signaling pathway has been shown to participate in various Drosophila developmental processes such as dorsal and thorax closure (Riesco-Escovar etal. 1996, Sluss et al. 1996, Agnes et al. 1999, Zeitlinger \& Bohmann 1999), planar polarity determination in eye and wing development (Paricio et al. 1999, Weber et al. 2000), control of morphogenetic apoptosis (Adachi-Yamada \& O'Connor 2002) and regulation of imaginal disc proliferation (Read et al. 2004). The involvement of JNK signaling in

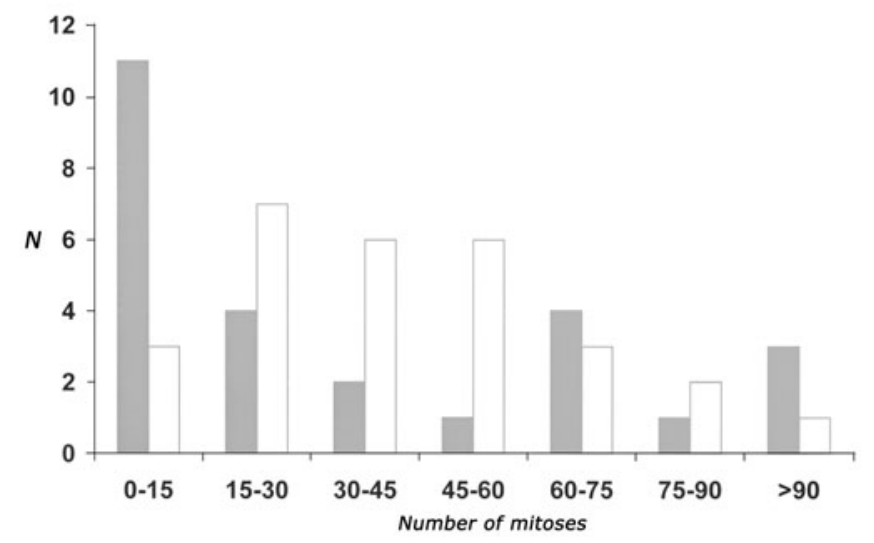

the wound healing of Drosophila larvae (Galko \& Krasnow 2004) and adult cuticle (Rämet et al. 2002) has been well established, suggesting that it might also play a role in imaginal disc wound healing. In this study, we address the question of how wound healing and initiation of regenerative proliferation are interconnected and in particular, how JNK signaling is related to these processes. We present evidence that JNK signaling participates in the imaginal disc wound healing and is regulated by the physical dynamics of the process. In addition, we show that the JNK cascade is an important part of the machinery recruiting cells into the regeneration process.

\section{Results}

\section{Time-lapse confocal imaging of incised GFP-moesin wing discs}

We have made an attempt to reveal the cell shape changes of the cut edge cells (hereafter referred to as leading edge cells), initially observed by Reinhardt \& Bryant (1981), during imaginal disc healing by utilizing live confocal imaging and in vitro culturing of incised discs (Fig. 1). We have employed a modified M3 medium (MM3) which was shown to support short time disc growth. The controls demonstrated that discs heal efficiently in vitro (data not shown) and further, continue to proliferate at least during the observed 16 hours (Fig. 2). The time-lapse confocal imaging of healing wing discs $(\mathrm{N}=10)$ from

Fig. 2. Comparison of number of mitosis between intact wing discs cultured for $\mathbf{1 6}$ hours in vivo and in vitro. As shown by Dale \& Bownes (1980), the cell divisions gradually decrease during the culture reflecting the conclusion of the disc's developmental programme. In the in vitro cultured discs (white bars), the average number of mitoses (42) coincide well with the results of Dale \& Bownes (1980), who found that after 1 day of in vivo cultivation on average 195 S-phase cells were present in the intact wing disc, constituting approximately 24 mitoses (estimate is based on the cell cycle phase duration; 4 hours for S-phase and 0.5 hours for mitoses). The in vivo cultured discs (grey bars) show a preferential distribution towards a low mitosis number (the average number of mitoses is 37). We expect it to be the result of a "cold shock", as the in vivo cultured discs were kept on ice before injection, mimicking the response to $X$ irradiation or heat shock followed by a transient suppression of proliferation. Ordinate, number of discs; Abscissa, number of mitoses in a given disc. 
sGMCA flies, constitutively expressing GFP-moesin fusion protein binding to actin (Kiehart et al. 2000), showed actin protrusions of 5 to $10 \mu \mathrm{m}$ long from the leading edge cells (Fig. 3). These structures closely resembled in size and form to the filopodia seen in embryonic wound healing (Wood etal.2002) as well as in dorsal closure (Jacinto et al. 2000). Filopodia were most abundant in the close proximity of the vertex of the healing wound and they were never seen to form further than $50 \mu \mathrm{m}$ away from the location of the apposed cut edges. These structures were first observed after 90 minutes of culturing. However, filopodia formation was rather a rare event, possibly reflecting limitations in the experimental procedures. Frequently, a mass of cell debris was seen to attach to the wound vertex making it impossible to discern the cell protrusions from the leading edge cells. In addition, although gently compressed between glass surfaces, the thickness of the imaginal discs $(30-25 \mu \mathrm{m})$ compelled us to use large intervals $(>1 \mu \mathrm{m})$ between optical sections. Consequently, some of the thin actin protrusions could have remained unnoticed.

In addition to the filopodia formation we observed a rapid reorganization of actin, within few minutes from the injury, into the presumed adherens junctions of the leading edge cells accompanied with cell elongation (Fig. 3). This "actin cable" persisted in some discs for as long as 10 hours in our culture conditions, whereas in some discs it was no longer visible after a few scans. We were not able to distinguish whether the re-organization of actin occurred in the cells of the peripodial membrane, columnar epithelia or both. As shown by Reinhardt \& Bryant (1981) the cells of the peripodial membrane and columnar epithelia curl towards each other, thus constituting socalled heterotypic contacts immediately after wounding. The close contact of the two cell layers might create difficulties in distinguishing between the actin concentrations of the different cell layers.

\section{Expression of puckered and misshapen in incised wing discs}

The role of the DJNK signaling in orchestrating cell shape changes during dorsal and thorax closure is well established (Riesgo-Escovar et al. 1996, Zeitlinger \& Bohmann 1999, Agnès et al. 1999). In order to study whether the pathway has a role in imaginal disc wound healing, we have taken advantage of the available DJNK cascade reporters, providing the possibility of visualizing active DJNK signaling with simple experimental procedures. The genes puckered (puc) and misshapen ( $m s n)$ are well known members of the DJNK cascade encoding a DJNK phosphatase and a Drosophila homologue of the Ste20 kinase (a MAPKKKK) acting upstream of DJNK, respectively (Martín-Blanco et al. 1997, Su et al. 1998).

The dissected late third instar imaginal wing discs from puc and $m s n$ enhancer-trap -strains were cut as shown in Fig. 1 (D'B cut) and cultured in vivo for $4,16,24$ or 48 hours. The expressions of puc and $m s n$ were found to coincide perfectly with the healing process, showing an intense $\beta$-galactosidase activity in the site of the induced wound. The overall dynamics of temporal and spatial expression are shown in Table 1, where the discs are classified according to their morphology and gene expression. The uncut controls, cultured for 24 hours, had occasional scattered stained cells without any preferential location, presumably representing sporadic injury produced by the injection process. Four hours of cultivation was sufficient to induce the gene expressions in approximately half of the examined discs (Table 1). The expressions were initially localized to the leading edge cells in both cell layers of the wing disc, i.e. the peripodial membrane and the columnar epithelia (Fig. 4A). Later in the process, in many of the discs the expression was clearly seen to extend laterally from the leading edge cells (Fig. 4B). This effect was especially evident in discs where healing was slow, i.e. in the few discs which due to variation in the wound closure process were still unhealed after 48 hours (see below), suggesting that prolonged existence of a wound leads to up-regulation of DJNK signaling in nearby cells. It is worth mentioning that in general the expression intensity of msn was low compared to that of puc (Fig. 4B and C). The effect might reflect the properties of the reporter insertions and was therefore disregarded. The expression of puc and msn remained localized to the wound until the healing process was completed. Culturing for 48 hours was sufficient to restitute continuous epithelia in nearly all of the discs resulting in subsequent downregulation of the DJNK activity (Table 1). It must be mentioned
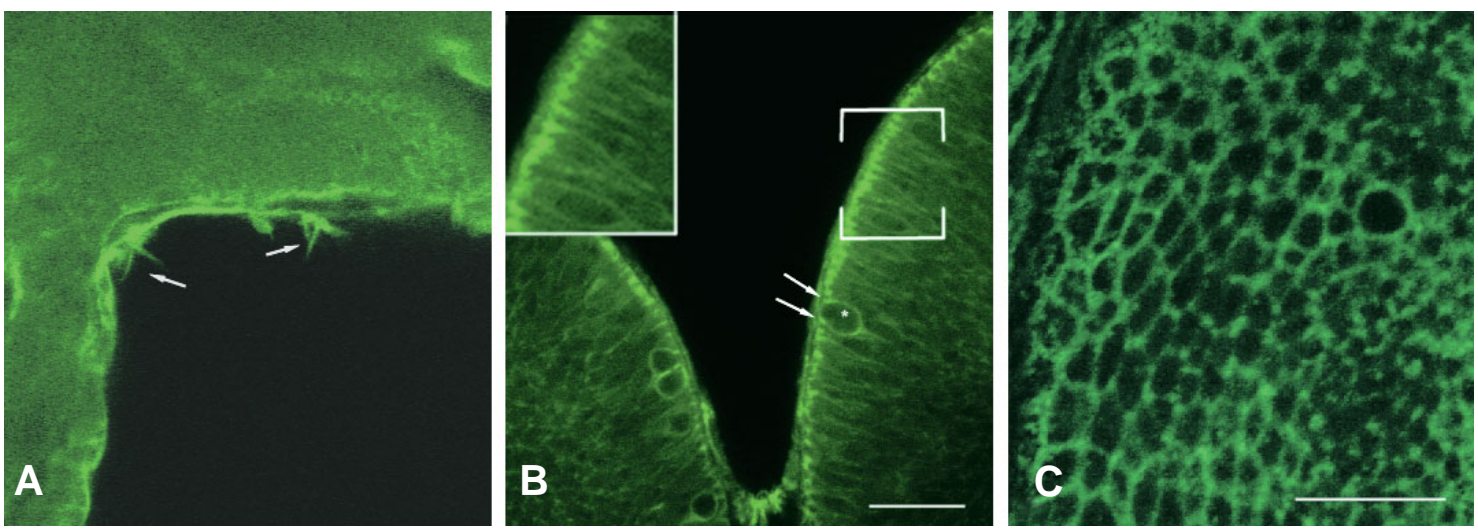

Fig. 3. Actin dynamics of a healing wing imaginal disc visualized by moesin-GFP (D'B cut). (A) A time lapse confocal image of a healing wing disc 90 minutes after the incision. The arrows point to filopodia protrusions from the leading edge cells. (B) A time lapse confocal image of a healing wing disc 10 minutes after the incision. A punctuate actin cable can be seen in the cells of the leading edge. A few large mitotic cells are also located in the cut edge and one of them, denoted by an asterisk, clearly shows moesin concentrations on the presumed adherens junctions (arrows). The area in brackets is enlarged to show the elongation of the cut edge cells. Scale bar, $20 \mu \mathrm{m}$. (C) An enlargement of the columnar epithelia of a non-cut and non-cultured wing disc showing the normal polygonal cell shapes. Scale bar, $10 \mu \mathrm{m}$. 

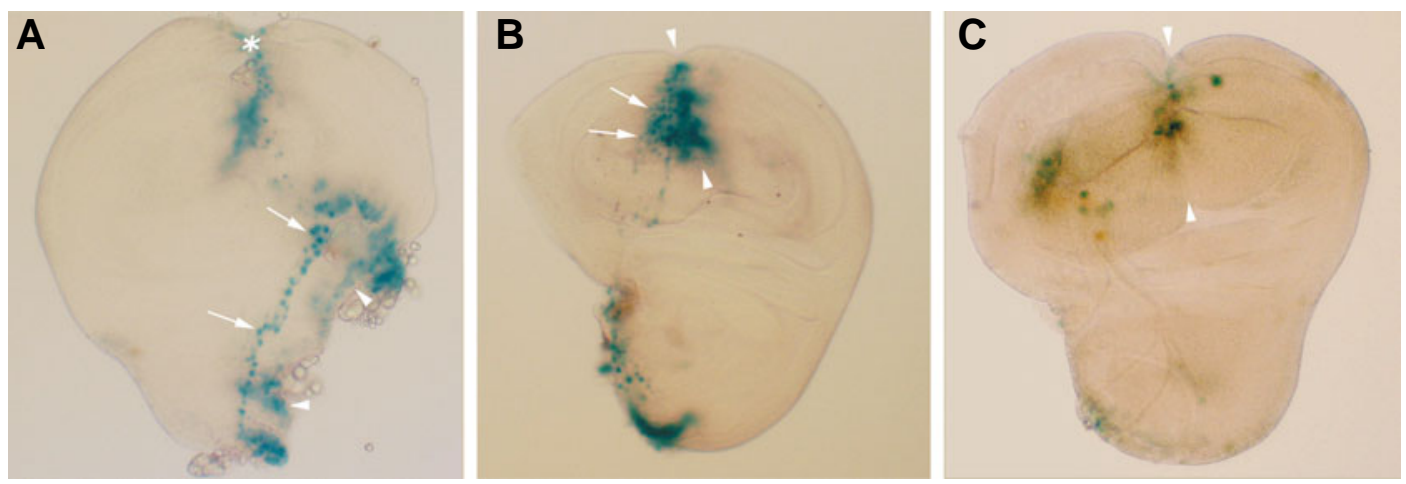

Fig. 4. Expression of puc and $\mathbf{m s n}$ during wound healing of a wing imaginal disc. (A) The expression of the puc gene is induced in the cells of the peripodial membrane and the columnar epithelia. An incised $\left(D^{\prime} B\right)$ wing disc after $16 \mathrm{~h}$ of in vivo cultivation. The asterisk denotes the original site of the incision. An accidental rupture in the notum demonstrates well the spatial expression pattern of the reporter; the stained nuclei are visible in the columnar epithelium (arrows) and the peripodial membrane (arrowheads, cell layer not in focus). (B) An incised (D'B) puc ${ }^{E 69}$ wing imaginal disc cultured for 24 hours in vivo. The expression of the puc gene can be seen to localize to the cells (arrows) several cell diameters from the site of the wound closure denoted by the arrowheads. The stained nuclei in the stalk region are peripodial and represent the normal expression pattern of puc E69 - strain in the late third instar larva. Anterior is to the left and dorsal up as in all the illustrated discs. (C) An incised (D'B) msn ${ }^{06946}$ wing imaginal disc cultured for 24 hours in vivo. The intensity of expression is significantly lower than in the puc E69 strain. Arrowheads denotes the site of the wound closure.

that the completion of the homotypic healing could not be recorded by simple light microscopy. Hence, we inferred the healing finished when the groove denoting the apposed wound edges was no longer visible.

The observed dynamics of the puc and msn expression suggested a dependency between the wound closure process and DJNK activity. To uncover this correlation we quantified the spatial gene expressions by measuring the area of the X-gal staining in open and closed wounds (the area of gene expression was divided by the area of the whole disc to sequester the effect of different cell numbers between discs). We found that the puc and $m s n$ expression negatively correlated with wound closure and further, that in open wounds the quantity of reporter expression increased significantly up to 16 hour time point and, if unhealed, remained high and localized to the cells of the leading edge and lateral to it (Fig. 5). We conclude that the induction of DJNK signaling in an imaginal disc is dependent on perturbation of the epithelia and further, that silencing of the pathway is the result of restitution of the continuous epithelia.

\section{Mitoses in a regenerating blastema}

To observe mitoses induced by the regeneration process we first cultured the whole discs for 4 days in vivo. This time period is sufficient to end the developmental proliferation of the disc and therefore reduce background mitoses significantly (Dale \& Bownes 1980). After recovering the discs from the hosts, they were cut according to Fig. 1 (A'B'C and D'B cuts) and reimplanted into fresh adult hosts. Subsequent to an additional culture period of 24,48

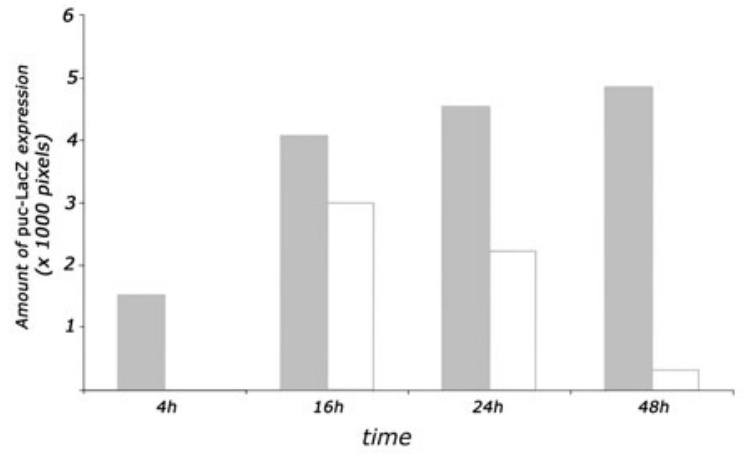

Fig. 5 (Left). Dynamics of puc expression in open and closed wounds

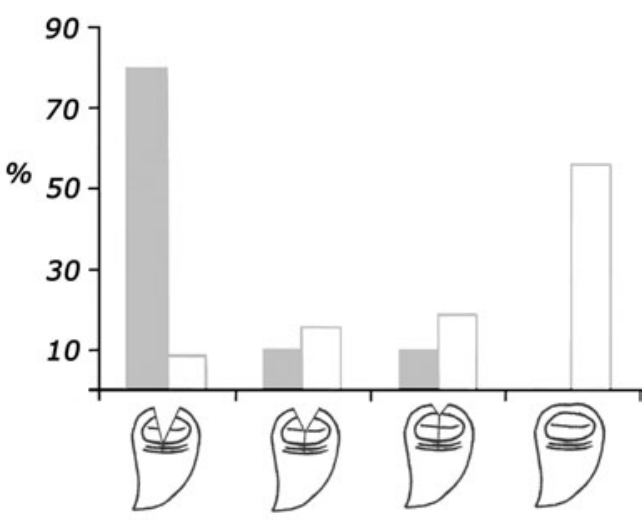

measuring the area (i.e. number of pixels) of stained cells in the wound. To sequester the difference in cell numbers between discs, the area of expression was divided by the whole area of a given disc. In open wounds (grey bars), the area of reporter expression increases until it reaches its maximum after 16 hours. After that the reporter expression persist in the wound and slightly increases at the later time points possibly reflecting the "spreading" of DJNK signal to cells lateral from the cut edge. In closed wounds (white bars), the area of expression decreases, thus reflecting the inhibitory role of the wound closure to the active DJNK signaling. Ordinate, the amount of reporter expression (stained area/total disc area); Abscissa, time.

Fig. 6 (Right). Comparison of the wound healing progression between wild type and 1782-GAL4;UAS-BskDN wing discs. The bars represent the proportion of wounded ( $D^{\prime} B$ cut) wild type (white bars, N=32) and 1782-GAL4;UAS-BskDN (grey bars, N=32) wing discs scored by morphology after 24 hours of in vivo cultivation. Nearly $80 \%$ of the BskDN wing discs are unhealed at this time, while the corresponding percentage in the wild type is less than 10. 
TABLE 1

\section{DYNAMICS OF PUCKERED AND MISSHAPEN EXPRESSION} DURING WOUND HEALING PROCESS

\begin{tabular}{|c|c|c|c|c|c|c|c|c|}
\hline \multirow[b]{3}{*}{ CLASS } & \multicolumn{8}{|c|}{$\%$ of discs $(\mathrm{N})$} \\
\hline & \multicolumn{4}{|c|}{ puckered } & \multicolumn{4}{|c|}{ misshapen } \\
\hline & Hrs 4 & 16 & 24 & 48 & 4 & 16 & 24 & 48 \\
\hline & $\begin{array}{l}38 \% \\
(16)\end{array}$ & - & - & - & $\begin{array}{l}53 \% \\
\text { (19) }\end{array}$ & - & - & - \\
\hline & $\begin{array}{l}62 \% \\
(16)\end{array}$ & $\begin{array}{r}9 \% \\
(22)\end{array}$ & - & $\begin{array}{l}8 \% \\
(12)\end{array}$ & $\begin{array}{l}47 \% \\
(19)\end{array}$ & $\begin{array}{l}17 \% \\
(12)\end{array}$ & $\begin{array}{l}14 \% \\
(14)\end{array}$ & - \\
\hline & & $\begin{array}{c}27 \% \\
(22)\end{array}$ & $\begin{array}{l}18 \% \\
(22)\end{array}$ & $\begin{array}{l}25 \% \\
(12)\end{array}$ & - & $\begin{array}{l}42 \% \\
(12)\end{array}$ & $\begin{array}{l}36 \% \\
(14)\end{array}$ & $\begin{array}{l}10 \% \\
(10)\end{array}$ \\
\hline & & $\begin{array}{l}64 \% \\
(22)\end{array}$ & $\begin{array}{l}82 \% \\
(22)\end{array}$ & - & - & $\begin{array}{l}42 \% \\
(12)\end{array}$ & $\begin{array}{l}50 \% \\
\text { (14) }\end{array}$ & $\begin{array}{l}10 \% \\
\text { (10) }\end{array}$ \\
\hline & - & - & - & $\begin{array}{l}67 \% \\
(12)\end{array}$ & - & - & - & $\begin{array}{l}80 \% \\
(10)\end{array}$ \\
\hline
\end{tabular}

or 72 hours, the discs were visualized for mitoses by labelling with $\mathrm{H} 3 \mathrm{p}$-antibody. We scored the number of mitoses in each blastema and also the localization of cell divisions with regard to the wound edges. First, we collated our findings with the already published results of proliferation in regenerating wing discs. Consistent with the observations of O'Brochta and Bryant (1987), we found that the average number of mitoses in a blastema of a straight cut control (D'B cut), cultured for 24 hours, was nearly identical compared to a fragmented disc (A'B'C cut) (Table 2). Further, we found no significant difference in the blastema size of the 24- and 48-hour cultured disc fragments (Table 2). However, in fragmented discs a noteworthy increase in the number of mitoses was observed by the third day of culture, resembling the data presented in O'Brochta and Bryant (1987) showing a rise in S-phase cells in the blastema between the first and second or second and third day in small and large cuts, respectively. As expected, we found that the overall number of mitoses in the blastema is significantly lower than the number of S-phase cells reported by O'Brochta and Bryant (1987). It is reasonable to believe that the difference is a consequence of the differential duration of S-phase and mitosis, mitosis being a significantly shorter event. Interestingly, there is evidence that the cell cycle duration in a regenerating blastema is not different from the cell cycle of a late wandering stage imaginal disc (Anne Sustar, personal communication). This means that one would expect to find a correlation between the number of S-phase and mitotic cells and the corresponding cell cycle stage duration. The Sphase of imaginal disc is reported to be about 4 hours (Reis \& Edgar 2004), being approximately eight times longer than mitosis. The number of S-phase cells in a $\mathrm{ZW}$ cut $\left(30^{\circ}\right.$ sector removed) in O'Brochta and Bryant (1987) is roughly 8 times higher than the mitoses counted in our experiment.

In order to gain additional information on mitosis induction in the blastema we utilized a dominant-negative form of Basket (Bsk) (Adachi-Yamada 1999), the Drosophila homologue of the mammalian JNK protein (Sluss et al. 1996, Riesco-Escovar et al. 1996). Our objective was to delay the process of wound healing by disrupting the JNK signaling and simultaneously record the effect on induction and localization of regenerative cell divisions. Overexpression of a dominant-negative form of bsk (bskDN), driven by an imaginal disc specific GAL4-driver (see material and methods), hampered the process of wound closure (Fig. 6). This can be interpreted as the consequence of blocking the JNK signaling pathway since BskDN has been shown to inhibit the JNK target puc and msn gene expressions in the larval cuticle (Galko \& Grasnow 2004). After 24 hours of in vivo culturing, $80 \%$ of the incised (B'D cut) wing discs expressing bskDN showed only little or no healing at all. The corresponding proportion in the wild type was less than $10 \%$ (Fig. 6). The distribution of wild type discs, seen in Fig. 6, demonstrates the large variation in the healing process. The effect can be partly explained by the injection procedure; as the discs are pulled into the capillary, the wound edges are sometimes apposed thus resulting in faster healing. On the contrary, the positioning inside the host fly may hinder the process, e.g. host tissue adhering to the disc can physically block wound contraction.

To test whether the utilized mutation has an influence on the normal developmental proliferation we compared the average number of mitoses between the wild type and BskDN late third instar wing discs and found no significant difference ( $t=1.29$, N.S.) (Fig. 7). The effect of DJNK disruption on the regenerative mitoses is shown in Fig. 7 and Table 2. Delayed wound healing did not result in concomitant hindrance of proliferation initiation. The blastema frequencies among the fragmented (A'B'C cut) WT discs were 47, 95 and 100 percent after $24(\mathrm{~N}=38), 48(\mathrm{~N}=26)$ and $72(\mathrm{~N}=6)$ hours of cultivation, respectively. After 48 hours of cultivation, blastema was present in 92 percent of BskDN disc fragments. This result is in agreement with the observations of Dale \& Bownes (1981), who showed that a physical barrier preventing the healing process does not delay the appearance of S-phase cells, indicating that the regulating cells recruited into the S-phase proceed to mitosis independently from the wound healing process. In addition, direct examination of the anti-pH3labelled discs clearly shows the localization of mitoses to the free wound edges of imaginal discs (Fig. 7). However, although unaltered in regard to the proliferation initiation and mitosis

TABLE 2

AVERAGE NUMBER OF MITOSES IN A REGENERATING BLASTEMA RESULTING FROM THE D'B AND A'B'C CUT IN WILD TYPE AND BSKDN WING IMAGINAL DISC

\begin{tabular}{lccc}
$\mathbf{t}$ & $\begin{array}{c}\text { WT } \\
\text { D'B }\end{array}$ & $\begin{array}{c}\text { WT } \\
\text { A'B'C }^{\prime}\end{array}$ & $\begin{array}{c}\text { Bsk-DN } \\
\text { A'B'C }^{\prime}\end{array}$ \\
\hline $\mathbf{2 4} \mathbf{~ h}$ & $20(11,2.6)$ & $22(17,2.9)$ & - \\
$\mathbf{4 8 ~ \mathbf { ~ h }}$ & - & $25^{*}(24,2.1)$ & $11^{*}(20,0.9)$ \\
$\mathbf{7 2} \mathbf{~ h}$ & - & $35(6,4.4)$ & - \\
\hline
\end{tabular}

In parenthesis is the number of cases and the standard error of the mean (SEM), respectively. *Student $t$-test value $t=6.07^{* * *}$ The dashes denote time points were mitoses were not recorded. 


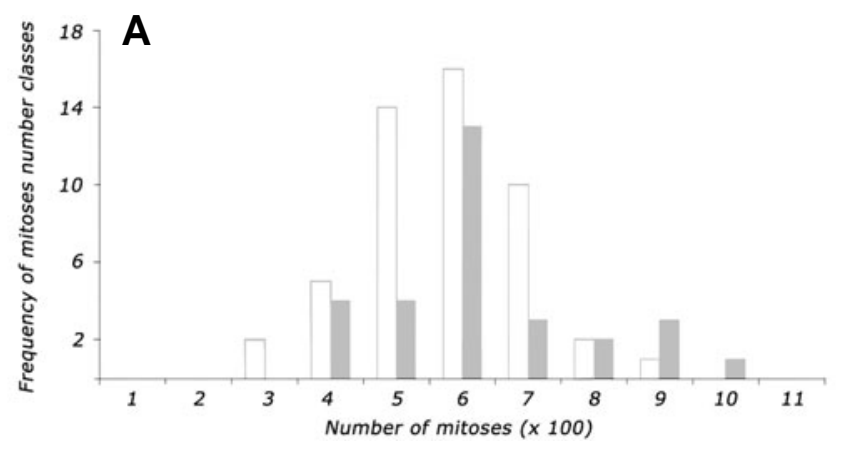

Fig. 7. BskDN effect on the proliferation in control non-cut and nontransplanted and in regenerating late third instar wing discs. (A) $A$ histogram showing the mitoses number distribution of wild type (white bars) and 1782-GAL4; UAS-BskDN (grey bars) control non-transplanted late third instar wing discs. We compared the average number of mitoses, 411 and 439 for wild type and 1782-GAL4; UAS-BSKDN, respectively and found it non-significant ( $t=1.29$ N.S.). The ordinate - frequency of the classes among the discs considered, The abscissa - mitoses number classes (mitoses number $x$ 100). (B) A regenerating wild type wing disc ( $A^{\prime} B^{\prime} C^{\prime}$ cut) cultured for 48 hours in vivo showing a mitosis concentration in the location of the extirpation. The wound has only partially healed and yet mitoses have occupied the area of the free wound edges (arrowheads). Mitoses are evident a few cell diameters away from the leading edge cells. (C) A regenerating wing disc $\left(A^{\prime} B^{\prime} C\right.$ cut) expressing the dominant negative form of bsk cultured for 48 hours in vivo. Only a few mitoses are located to the site of the extirpation (arrowhead). Similarly to the wild type, mitoses are seen in the area of the unhealed wound edges. Both discs have been cultured for 4 days in vivo prior to cutting. Scale bar, $50 \mu \mathrm{m}$.

localization, the number of cell divisions in a blastema of a BskDN wing disc was significantly reduced compared to the wild type (Table 2) $\left(t=6.07^{* * *}\right)$. This result suggests a requirement of JNK signaling in the regulation of blastema size in a regenerating wing disc. Taken together, we infer that these observations reflect the involvement of DJNK signaling specifically in the regulation of the regenerative proliferation.

\section{Discussion}

In this study, we address the question of how wound healing and commencement of regenerative proliferation in an imaginal disc are interconnected. We have shown that the imaginal disc healing process is accompanied by cell shape changes and actin accumulation in the leading edge cells of the wound. Our results also demonstrate the activation and requirement of JNK signaling in the wound healing process. In addition, we have been able to address a role of JNK in the regulation of the blastema size of a regenerating wing disc, suggesting the existence of a mutual signaling event for wound healing and initiation of regeneration.

\section{Imaginal disc wound healing}

In our live confocal imaging of healing GFP-moesin wing discs, we found a rapid formation of a punctuate actin cable to the leading edge cells. Such an actin cable has been reported to play a role in the morphogenesis and wound healing of Drosophila embryos (Young et al. 1993, Wood et al. 2002), in injured singlecelled Xenopus oocytes (Bement et al. 1999), in the healing of chick embryo epidermises (Martin \& Lewis 1992) as well as in the repair of tissue culture wounds (Bement et al. 1993, Altan \& Fenteany 2004). It has been suggested that the actin cable functions as a "purse string" contracting the wound edges together (reviewed by Kiehart 1999). We hypothesize that the observed actin cable has a similar role in imaginal disc wound healing.
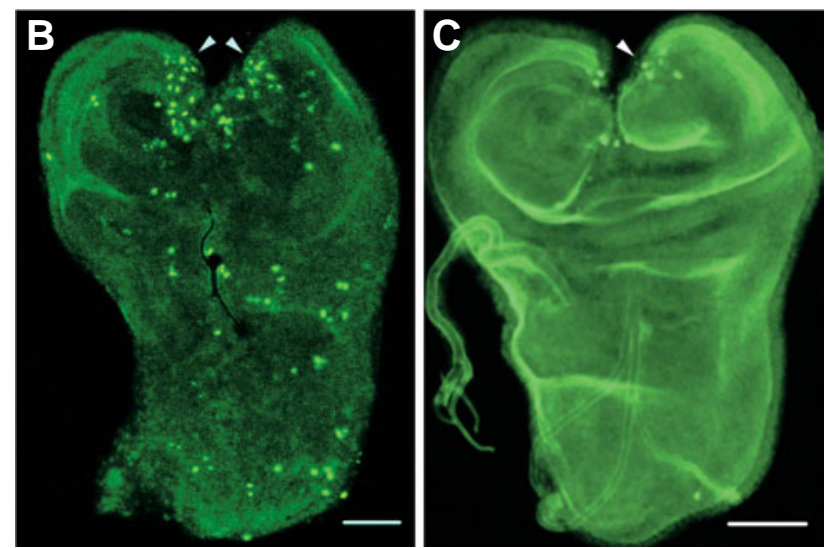

In dorsal closure, filopodial activity has been assigned to epithelial adhesion and proper cell alignment (Jacinto et al. 2000). Filopodia have also been shown to form and interdigitate during the late stages of imaginal disc wound healing (Reinhardt et al. 1977, Reinhardt \& Bryant 1981). Here we have shown that the first actin-rich cell protrusions extend from the leading edge cells approximately 90 minutes after the wounding. By comparison, filopodia form within minutes of wounding in Drosophila embryos (Wood et al. 2002) and within several hours (4 hours) in adult cuticle epidermis (Rämet et al. 2002). Taken together, these results demonstrate a gradual temporal decline from an embryonic to a terminally differentiated cell's ability in the rapidity to reorganize the cytoskeleton as a response to wounding.

In a recent study by Altan and Fenteany (2004), the authors show that JNK signaling regulates lamellipodial protrusion in wounded Madin-Darby canine kidney cell cultures by gene expression-independent mechanisms. Regardless of the finding that disruption of the DJNK cascade by BskDN significantly hinders the process of imaginal disc healing, the specific proof that JNK signaling actually regulates the cell morphology in imaginal disc wound healing is still to be elucidated. However, it can be concluded from the rapid changes in actin cytoskeleton observed in our experimental system that these events are also mediated by a gene expression-independent cytoplasmic transducer. Our method of in vitro cultivation together with time lapse confocal imaging will enable future mutational studies of the process.

\section{The role of JNK signaling}

The activation of the DJNK signaling cascade is a rapid reaction to the incision of the imaginal disc. Only after 4 hours of in vivo cultivation can the DJNK responsive gene-expression, visualized by puckered-LacZ, be seen to localize to the leading edge cells of the wing disc fragment. Our finding closely correlates with the results of Rämet et al. (2002) who observed the up- 
regulation of puc expression in the adult epidermis after just three hours of wound induction. Our data also demonstrate that the silencing of the DJNK pathway is connected to the restitution of the continuous epithelia and further that the prolonged existence of a wound results in elevated reporter expression possibly reflecting the up-regulation of the pathway in nearby cells. Interestingly, in adult epidermis wound healing, DJNK activity was seen to extend several rows lateral from the leading edge and the intensity of extension positively correlated with the size of the wound, i.e. the time required for re-epithelization (Rämet et al. 2002). Similarly, in a recent study by Galko \& Krasnow (2004), it was shown that during larval cuticle wound healing the DJNK signaling is induced in a gradient emanating from the wound edges out to at least five cell diameters away. Based on these results, we suggest the existence of autoactivation of the DJNK cascade mediated by the discontinuous epithelia. How the signal emanates from the leading edge to more lateral cells is unknown.

Similar to Bryant \& Fraser (1988), we first cultured the discs in vivo in order to halt the developmental proliferation, leaving the cells in a mitotically quiescent phase. To commence regeneration, the cells near the cut edge have to be recruited back into the cell cycle (Adler 1984). It is known that after an extensive proliferation during larval development the disc halts its growth; at $0 \mathrm{~h}$ APF most of the cells in the wing blade are arrested in G2 (Milan et al. 1996), waiting for the hormonal cue to begin an additional round of mitoses and the morphogenetic movements leading to wing formation. Conversely, inconsistent with the normal disc development, a protracted in vivo culturing of an intact wing disc results in a predominant proportion of $\mathrm{G} 1$ cells $(55 \%$, data from Fain \& Alvarez, 1987). Although there is no direct proof to demonstrate whether the cells participating in the regeneration process are initially in $\mathrm{G} 2$ or G1/G0, evidence exists in favour of the latter. In Fain \& Alvarez (1987), a significant increase in G2 cells was observed in dissociated and reaggregated imaginal disc cells cultivated in vivo for 12 hours. In addition, cultured imaginal disc fragments show a lag period of at least 12 hours before an increase in cell number is evident, suggesting that S-phase cells appear first (Adler 1981, Kiehle \& Schubiger 1985) and further, comparison of blastema frequencies presented here (blastema present in 47\% of the discs after 24 hours) and in O'Brochta \& Bryant (1987) demonstrate the earlier appearance of blastemas visualized with $S$-phase cells.

Recently, Goss et al. (2003) have observed that in HeLa cells JNK regulates the cdc2/cyclin $B$ complex by phosphorylating serine 168 of cdc25 as a response to cell stress leading to G2/M arrest. In our experiment, BskDN was shown to inhibit mitoses in the regenerating blastema, thus suggesting that although JNK has a role of inhibiting cell cycle under certain conditions, as in response to cell stress, it also has an indirect function, via gene expression, in recruiting quiescent cells into the cell cycle. Interestingly, Pedraza et al. (2004) have shown that, when ectopically expressed, the Src64 and Src42 kinases, Drosophila members of the Src-family kinases (SFKs), are able to recruit $\mathrm{G} 1 / \mathrm{G} 0$ cells back into the cell cycle in a developing eye disc. SRCs are known oncogenic proteins, which regulate in addition to cellular proliferation, the cell's adhesion and motility by disassembling the cell's focal adhesions and adherens junctions (see Yeatman 2004 for review). Drosophila Src42A is known to play a role in dorsal closure by directly phosphorylating Bsk (Tateno et al. 2000), implying a possible connection between SRCs and Bsk in recruiting quiescent cells back into the cell cycle in a regenerating imaginal disc. Concomitant with these observations, Read et al. (2004) have genetically shown the interaction between DJNK signaling and SRCs in the regulation of eye-antennal disc proliferation.

\section{Wound healing and regenerative proliferation}

We have shown directly for the first time that mitoses precede the homotypic healing of the cut edges in imaginal disc regeneration. Our result confirms the observations of Kiehle \& Schubiger (1985) who reported a rise in mitotic index near the wound edges before wound closure and Abbot et al. (1981), who found that clone frequencies were highest when induced at the time of fragmentation. Taken together, these observations evidently revoke the proposed requirement of homotypic healing prior to the initiation of regenerative cell divisions. Consequently, these results and the finding that BskDN suppresses the regenerative cell divisions, lead to the suggestion that any cut, including a hypothetical situation where all cells are left viable, will initiate proliferation via the DJNK pathway. Abbot et al. (1981) have shown that clones induced later in the regeneration process were preferentially located in the newly formed tissue, suggesting that the later dividing cells are the progeny of the initially recruited cells. Such a dynamic could therefore reflect the activity of DJNK signaling; after the pathway has ceased no more cells would be recruited from the "old" tissue to enter the regeneration process. In addition, our results demonstrate that DJNK signaling is sometimes upregulated in cells lateral to the leading edge, suggesting that the signal to proliferate could extend to nearby tissue. Interestingly, Adler (1984) has reported a propagation of the proliferation stimulus to cells away from the cut edge when culturing a fragment containing the prospective wing proper.

Since proliferation is known to continue long after fusion of the wound edges (O'Brochta \& Bryant 1987), other mechanisms controlling the longevity of a blastema, reflecting a "proliferation memory", have to play a role. Interestingly, in our experiment an increase in mitosis number between the second and third day was observed. A similar "jump" in the number of dividing cells is also apparent from the data of Dunne (1981) and O'Brochta and Bryant (1987). Temporally, this event takes place after the wound healing, suggesting that cell-cell interactions are enhancing the proliferation rate in these cases. In O'Brochta \& Bryant (1987) it is even evident that in a larger cut the rise takes place later than in smaller cuts, possibly reflecting the differential duration of the healing process. These findings lead us to hypothesize that the cell divisions in a blastema are regulated in a two-step process. (1) The longer the wound is healing, the more cells DJNK cascade is recruiting to the cell cycle. Since the pace of imaginal disc healing is dependent on the morphology of the fragment, i.e. fragments with large wounds heal slower than fragments with small wounds (Reinhardt et al. 1977), in larger cuts this would lead to an initially larger number of regulating cells. Consequently, after the DJNK signaling has ceased, no more cells are entering into the cell cycle by this mechanism. (2) After the apposition of the wound edges, the already dividing cells will "decide" whether to divide again based on the discontinuity in positional gradient. As seen from the data of O'Brochta and Bryant (1987), the increase in blastema size correlates with the amount of tissue 
removed. This kind of growth dynamics suggests a role for "the positional value discrepancy"; the steeper the gradient between the apposed cells, the higher is the probability of a cell to divide per time unit.

\section{Materials and Methods}

\section{Fly stocks}

We used Oregon RS as a wild type strain. The actin dynamics of the healing wing imaginal discs were visualized in $w$; sGMCA line (Kiehart $e t$ al. 2000) obtained by courtesy of Dr. Kiehart. In this line, a moesin-GFP fusion protein binding to actin is constitutively expressed in the fly epidermis. The activity of the JNK pathway was detected by misshapen and puckered enhancer traps; puc E69 (Ring \& Martinez Ariaz 1993) and $\mathrm{P}\left\{r y^{+t 7.2}=\mathrm{PZ}\right\} m s n^{06946} r y^{506} / \mathrm{TM} 3, r r^{R K} \mathrm{Sb}^{1} \mathrm{Ser}^{1}$ (Bloomington 11707). To detect the effect of JNK mutant in wound healing and regenerative proliferation a dominant negative form of Basket (BskDN) was specifically induced in imaginal disc cells by utilizing the UAS-GAL4 method (Brand \& Perrimon 1993). $w^{1118} \mathrm{P}\left\{w^{+m C}=\mathrm{UAS}-b s k\right.$.DN $\} 2$ (Bloomington 6409) females were crossed with $w^{*} ; \mathrm{P}\left\{w^{+m w} . h s=\right.$ Gaw $\} 32 \mathrm{~B}$ (Bloomington 1782) males and the resulting third instar larvae were collected for the experiments. The expression of the used GAL4-driver was tested with UAS-GFP reporter and was found to induce strong GFP expression in the wing proper.

\section{Culturing and time lapse confocal imaging of imaginal discs}

The discs were cut according to the Fig. 1 using a fine tungsten needle. In the healing experiments, i.e. time lapse confocal imaging and observation of the dynamics of puc-LacZ and msn-LacZ expression, an incisional cut along the midline of the wing proper was used, hereafter referred to as D'B cut. In the experiments considering regenerative proliferation, a wedge shaped extirpation, approximately $30^{\circ}$ in angle, was used, referred to as $A^{\prime} B$ ' $C$ cut. In the latter experiments, the D'B cut was also utilized to compare the proliferative stimulus of straight cut and tissue extirpation. We have utilized in vivo and in vitro culturing of imaginal discs. The in vivo culturing was performed as described in Mattila et al. (2004). In all the in vivo culture experiments the dissected wing discs from the late third instar were kept on ice before injection. Time lapse confocal imaging of healing wing discs was performed in Shields and Sang M3 insect medium (Sigma) supplemented with $260 \mathrm{mg} / \mathrm{l} \mathrm{KCl}, 3 \mathrm{mg} / \mathrm{l}$ cholesterol, $50 \mathrm{\mu l} / \mathrm{ml}$ fly extract, $0.125 \mathrm{IU} / \mathrm{ml}$ insulin, $100 \mathrm{U} / \mathrm{ml}$ penicillin, $0.1 \mathrm{mg} / \mathrm{ml}$ streptomycin and $2 \%$ FCS. The M3 medium was prepared according to the manufacture's instructions. During the confocal imaging, the discs were kept at room temperature in $5 \% \mathrm{CO}_{2}$. The in vitro control experiments were performed at $25^{\circ} \mathrm{C}$ in $5 \% \mathrm{CO}_{2}$. We found that in the described culture medium, the imaginal discs lose their morphology after some 5-6 hours of cultivation, becoming spherical in shape. Despite attempts to optimize our medium, we were unable to prevent this effect. However, we found that by placing a small piece of cover slip (approximately twice the area of the imaginal disc) above the disc it was possible to prevent the change in morphology and yet obtain perfect healing. By doing so, we were also able to immobilize the disc on the bottom of a culture dish enabling the time lapse confocal imaging. In the time lapse experiments, the discs were monitored for 16 hours and scanned at one hour intervals. It must be noted that the healing process was slower compared to the control discs cultivated in an incubator, suggesting that the laser beam has an inhibitory effect upon the process. 16 hours was not sufficient to complete closure of the wound in the conditions of live cytology. All confocal images were obtained by a Zeiss LSM 510 META laser scanning confocal microscope.

\section{Visualization of gene expression and immunocytochemistry}

The $m s n-L a c Z$ and puc-Lac $Z$ expression was visualized with a $\beta$-Gal staining kit (Roche) using the protocol provided by the manufacturer. Stained discs were mounted in PBS and photographed with an Olympus DP11 digital camera attached to an Olympus BH-2 light microscope.

The H3-p staining of wing discs was performed with a H3-p primary antibody (rabbit anti-H3-p [Upstate Biotechnology], 1:100 dilution). The following secondary antibody was FITC conjugated goat-anti-rabbit IgG (Molecular Probes, 1:200 dilution). The discs were mounted in Mowiol supplemented with $10 \% \mathrm{DABCO}$ and $0.2 \% \mathrm{NaN}_{3}$ and photographed with a Leitz Wild MPS46 camera attached to a Leitz Dialux 22 fluorescence microscope. The pictures obtained were digitally processed using the Adobe Photoshop 5.5 software.

\section{Blastema measurement}

In our experiments, the in vivo pre-culture of the discs significantly reduced the background mitoses and allowed specific observation of the regenerative proliferation. However, the injection procedure may cause arbitrary damage in the wing proper, e.g. if the disc adheres to the class capillary, resulting in mitoses not induced by the cut. To sequester this effect from the material we decided to scale the areas from which mitoses were recorded. In each disc, the width of the wing proper was measured and divided by three. This measurement was considered to be the blastema width and mitoses inside it were scored. In the case of unhealed or partially healed wounds, we used a distance of $30 \mu \mathrm{m}$ from the free wound edges to determine the area from which regenerative mitoses were counted. To consider blastema present, i.e. regenerative proliferation initiated, we required a minimum of five mitoses inside the defined area. The number of mitoses was directly counted from the immunostained whole mount imaginal discs.

\section{Acknowledgements}

This work has been supported by the University foundation of Turku and by the joint agreement of the Academy of Finland and the Russian Academy of Science (joint project N6) and RFBR 05-04-48316.

\section{References}

ABBOT, L.C., KARPEN, G.H. and SCHUBIGER, G. (1981). Compartmental restrictions and blastema formation during pattern regulation in Drosophila imaginal leg discs. Dev Biol 87:64-75

ADACHI-YAMADA, T., NAKAMURA, M., IRIE, K., TOMOYASU, Y., SANO, Y. MORI, E., GOTO, S., UENO, N., NISHIDA, Y. and MATSUMOTO, K. (1999). P38 mitogen-activated protein kinase can be involved in transforming growth factor $\beta$ superfamily signal transduction in Drosophila wing morphogenesis. Mol Cell Biol 19:2322-2329

ADACHI-YAMADA, T. and O'CONNOR, M.B. (2002). Morphogenetic apoptosis: A mechanism for correcting discontinuities in morphogen gradients. Dev Biol 251:74-90

ADLER, P.N. (1981). Growth during pattern regulation in imaginal discs. Dev Biol 87:356-373

ADLER, P.N. (1984). DNA replication and pattern regulation in the imaginal wing disc of Drosophila. Dev Biol 102:300-308

AGNES, F., SUZANNE, M. and NOSELLI, S. (1999). The Drosophila JNK pathway controls the morphogenesis of imaginal discs during metamorphosis. Development 126:5453-5462

ALTAN, Z. M. and FENTEANY, G. (2004). c-Jun N-terminal Kinase regulates lamellipodial protrusion and cell sheet migration during epithelial wound closure by a gene expression-independent mechanism. Biochem Biophys Res Commun 322:56-67

BEMENT, W.M., FORSCHER, P. and MOOSEKER, M.S. (1993). A novel cytoskeletal structure involved in purse string wound closure and cell polarity maintenance. J Cell Bio/ 121:565-578

BEMENT, W.M., MANDATO, C.A. and KIRSCH, M.N. (1999). Wound-induced assembly and closure of an actomyosin purse string in Xenopus oocytes. Curr Biol 9:579-587.

BRAND, A.H. and PERRIMON, N. (1993). Targeted gene expression as a means of altering cell fates and generating dominant phenotypes. Development 118:40115.

BRYANT, P.J. (1971). Regeneration and duplication following operations in situ on 
the imaginal discs of Drosophila melanogaster. Dev Biol 26:606-615

BRYANT, P.J. and FRASER, S.E. (1988). Wound healing, cell communication and DNA synthesis during imaginal disc regeneration in Drosophila. Dev Biol 127:197-208

CHEN, Y. and STRUHL, G. (1996). Dual roles for patched in sequestering and transducing hedgehog. Cell 87:553-563

DALE, L. and BOWNES, M. (1980). Is regeneration in Drosophila the result of epimorphic regulation? Wilhelm Roux's Archives 189:91-96

DALE, L. and BOWNES, M. (1981). Wound healing and regeneration in the imaginal wing disc of Drosophila. Wilhelm Roux's Archives 190:185-190

DUNNE, J.F. (1981). Growth dynamics in the regeneration of a fragment of the wing imaginal disc of Drosophila melanogaster. Dev Biol 87:379-382

FAIN, M.J. and ALVAREZ, C. (1987). The cell cycle and its relation to growth during pattern regulation in wing discs of Drosophila. J Insect Physiol 33:697-705

FRENCH, V., BRYANT, P.J. and BRYANT, S.V. (1976). Pattern regulation in epimorphic fields. Science 193:969-981

GALKO, M.J. and KRASNOW, M.A. (2004). Cellular and genetic analysis of wound healing in Drosophila larvae. PLOS Biol 2(8):e239

GIBSON, M.C. and SCHUBIGER, G. (1999). Hedgehog is required for activation of engrailed during regeneration of fragmented Drosophila imaginal discs. Development 126:1591-1599

GOSS, V.L., CROSS, J.V., MA, K., QIAN, Y., MOLA, P.W. and TEMPLETON, D.J. (2003). SAPK/JNK regulates cdc2/cyclin B kinase through phosphorylation and inhibition of cdc25c. Cell Signal 15:709-718

HELD, L.I. JR. (2002). Imaginal discs: Cellular and genetic logic of pattern formation. University press, Cambridge, pp.152-155

JACINTO, A., WOOD, W., BALAYO, T., TURMAINE, M., MARTINEZ-ARIAS, A. and MARTIN, P. (2000). Dynamic actin-based epithelial adhesion and cell matching during Drosophila dorsal closure. Curr Biol 10:1420-1426

KIEHART, D.P. (1999). Wound healing: The power of the purse string. Curr Bio 9:R602-605

KIEHART, D.P., GALBRAITH, C.G., EDWARDS, K.A., RICKOLL, W.L. and MONTAGUE, R.A. (2000). Multiple forces contribute to cell sheet morphogenesis for dorsal closure in Drosophila. J Cell Biol 149:471-490

KIEHLE, C.P. and SCHUBIGER, G. (1985). Cell proliferation changes during pattern regulation in imaginal leg discs of Drosophila melanogaster. Dev Biol 109:336-346

MARTIN, P. and LEWIS, J. (1992). Actin cables and epidermal movement in embryonic wound healing. Nature 360:179-183

MARTÍN-BLANCO, E., GAMPEL, A., RING, J., VIRDEE, K., KIROV, N., TOLKOVSKY, A.M. and MARTINEZ-ARIAS, A. (1997). Puckered encodes a phosphatase that mediates a feedback loop regulating JNK activity during dorsal closure in Drosophila. Genes Dev 12:557-570

MATTILA, J., OMELYANCHUK, L. and NOKKALA, S. (2004). Dynamics of decapentaplegic expression during regeneration of the Drosophila melanogaster wing imaginal disc. Int J Dev Biol 48:343-347

MEINHARDT, H. (1983). Cell determination boundaries as organizing regions for secondary embryonic fields. Dev Bio/ 96:375-385

MILÁN, M., CAMPUZANO, S. and GARCæA-BELLIDO, A. (1996). Cell cycling and patterned cell proliferation in the Drosophila wing during metamorphosis. Proc Nat/ Acad Sci 93:11687-11692

O'BROCHTA, D.A. and BRYANT, P.J. (1987). Distribution of S-phase cells during the regeneration of Drosophila imaginal wing discs. Dev Biol 119:137-142

PARICIO, N., FEIGUIN, F., BOUTROS, M., EATON, S. and MLODZIK, M. (1999). The Drosophila STE20-like kinase Misshapen is required downstream of the Frizzled receptor in planar polarity signaling. EMBO J 18:4669-4676
PEDRAZA, L.G., STEWART, R.A., LI, D. and XU, T. (2004). Drosophila Src-family kinases function with $\mathrm{Csk}$ to regulate cell proliferation and apoptosis. Oncogene 23:4754-4762

READ, R.D., BACH, E.A. and CAGAN, R.L. (2004). Drosophila C-terminal src kinase negatively regulates organ growth and cell proliferation through inhibition of the src, jun N-terminal kinase and STAT pathways. Mo/ Cel/ Bio/24:66766689

REINHARDT, C.A., HODGKIN, N.M. and BRYANT, P.J. (1977). Wound healing in the imaginal discs of Drosophila I. Scanning electron microscopy of normal and healing wing discs. Dev Biol 60:238-257

REINHARDT, C.A. and BRYANT, P.J. (1981). Wound healing in the imaginal discs of Drosophila II. Transmission electron microscopy of normal and healing wing discs. J Exp Zool 216:45-61

REIS, T. and EDGAR, B.A. (2004). Negative regulation of dE2F1 by cyclindependent kinases controls cell cycle timing. Cell 117:253-264

RIESGO-ESCOVAR, J.R., JENNI, M., FRITZ, A. and HAFEN, E. (1996). The Drosophila Jun-N-terminal kinase is required for cell morphogenesis but not for DJun-dependent cell fate specification in the eye. Genes Dev 10:2759-2768

RING, J.M. and MARTINEZ ARIAS, A. (1993). puckered, a gene involved in position-specific cell differentiation in the dorsal epidermis of the Drosophila larva. Dev Supp/ 251-9

RÄMET, M., LANOT, R., ZACHARY, D. and MANFRUELLI, P. (2002). JNK signaling pathway is required for efficient wound healing in Drosophila. Dev Biol 241:145-156

SCHUBIGER, G. (1971). Regeneration, duplication and transdetermination in fragments of the leg disc of Drosophila. Dev Biol 26:277-295

SCHUBIGER, G. (1973). Regeneration of Drosophila melanogaster male leg disc fragments in sugar fed female hosts. Experientia 29:631-632

SLUSS, H.K., HAN, Z., BARRETT, T., DAVIS, R.J. and IP, Y.T. (1996). A JNK signal transduction pathway that mediates morphogenesis and an immune response in Drosophila. Genes Dev 10:2745-2758

SU, Y.C., TREISMAN, J.E. and SKOLNIK, E.Y. (1998). The Drosophila Ste20related kinase misshapen is required for embryonic dorsal closure and acts through a JNK MAPK module on an evolutionarily conserved signaling pathway. Genes Dev 12:2371-2380

TATENO, M., NISHIDA, Y. and ADACHI-YAMADA, T. (2000). Regulation of JNK by src during Drosophila development. Science 287:324-327

WEBER, U., PARICIO, N. and MLODZIK, M. (2000). Jun mediates Frizzled-induced R3/R4 cell fate distinction and planar polarity determination in the Drosophila eye. Development 127:3619-29

WOOD, W., JACINTO, A., GROSE, R., WOOLNER, S., GALE, J., WILSON, C. and MARTIN, P. (2002). Wound healing recapitulates morphogenesis in Drosophila embryos. Nat Cel/ Biol 4:907-912

YEATMANN, T.J. (2004). A renaissance for src. Nat Rev Cancer 4:470-480

YOUNG, P.E., RICHMAN, A.M., KETCHUM, A.S. and KIEHART, D.P. (1993). Morphogenesis in Drosophila requires nonmuscle myosin heavy chain function. Genes Dev 7:29-41

ZEITLINGER, J. and BOHMANN, D. (1999). Thorax closure in Drosophila: involvement of Fos and the JNK pathway. Development 126:3947-56

Received: January 2005 Reviewed by Referees: February 2005 Modified by Authors and Accepted for Publication: March 2005 Edited by: Christine Mummery 